\title{
Research, Development and Application of the Training Device for Trouble Shooting of Secondary Circuit of Operating Mechanism
}

\author{
Zhang $\mathrm{Su}^{1, \mathrm{a}}$, Zhu Yuanda ${ }^{1, \mathrm{a}}$, Ge Jun ${ }^{2, \mathrm{~b}}$, Zheng Wanyi ${ }^{1, \mathrm{a}}$, Shan $\mathrm{Jia}^{3, \mathrm{c}}$ \\ ${ }^{1}$ Liaoning Skill Training Center \\ ${ }^{2}$ Benxi Power Supply Company, State Grid Liaoning Electric Power Supply Co., Ltd. \\ ${ }^{3}$ Fushun Power Supply Company, State Grid Liaoning Electric Power Supply Co., Ltd. \\ a22181221@qq.com, bxwl_650928@126.com, ${ }^{\text {c } 105442377 @ q q . c o m ~}$
}

Keywords: breaker; secondary circuit; fault finding

\begin{abstract}
Fault simulation is an important part of power system dynamic simulation, fault models in fully dynamic simulation software of power system simulates all kinds of symmetric and asymmetric complex faults by fault branch admittance matrix. Handling multiple faults and random faults by admittance matrix shrinkage technology can take care of any number of faults in one branch and does not need to add branches or nodes, improving efficiency and using convenience. This paper introduces a fault simulation training equipment box of secondary circuit breaker, i.e. secondary circuit fault finding training equipment of operating system that is suitable for training and teaching in electric power applications.
\end{abstract}

\section{Introduction}

Secondary circuit fault clearing in electrical equipment is always a basic skill for electrical staff to grasp and also an important part in stuff training, evaluation, competition and other projects. Currently, training process for secondary circuit fault clearing of operating system is started from setting man-made faults and then requiring the trained to find out and clear the faults. Faults are traditionally set specifically by pasting tap as insulation on contact point of corresponding fault contact terminal and then reinstall it as the fault. This method takes much effort and time and frequent disassembly of wires may lead to loose screws on the contact, resulting in exposed wires and the system unable to get power support. This can only fixed by changing contactors and the process is time-consuming. In addition, since many nodes on the main loop of the operating system are crimped or plugged in, it is impossible to set man-made faults there. Much time would be wasted on theoretical analysis and schematic explanations as no actual test items is available for students to do measurement. Therefore, many faults finding can not be trained in the process of teaching and thus the training effect is not ideal. This paper introduces a fault simulation training equipment box of secondary circuit breaker, i.e. secondary circuit fault finding training equipment of operating system that is suitable for training and teaching in electric power applications.

\section{Schematic}

The first wire part connected with the secondary circuit is plotted as three lines and the rest are expressed in one line. Schematic is mostly used to learn or analyze the principle of protection devices and automatic device or original basis for the design of secondary circuit.

A, Instruments and relays of the schematic are in the overall form of equipment graphical notations but with connected contacts, instead of internal circuit diagram.

$\mathrm{B}$, Schematics draws the parts of the secondary current loop, voltage loop, DC circuit and the first circuit together to make readers have a complete concept over the constitution of the entire apparatus and also understand clearly the electrical connection and operation principle between all devices. 
C, Disadvantages: some details of the second terminal are not complete and have no internal wiring of components. Only part of the terminal block number, loop number and wire are presented and only the polarity of DC power supply is marker out.

Unfolded view: unfolded view and schematic are two expressions of the same wiring. The unfolded view is more explicit. As shown in Fig. 1:

A, The secondary circuit device is expanded into AC current, AC voltage circuit, DC circuit and signal circuit.

B, Different devices are connected according to the requirements of circuit to form separate circuits.

C, Coils and contacts of the same device (electrical component) is represented as the same text symbol. Similar devices are represented as numbers if there are many.

$\mathrm{D}$, There are explanations about the use of the circuit on the right of the map.

$\mathrm{E}$, All contacts of the components in the unfolded view are represented normally, that is to say no movements.

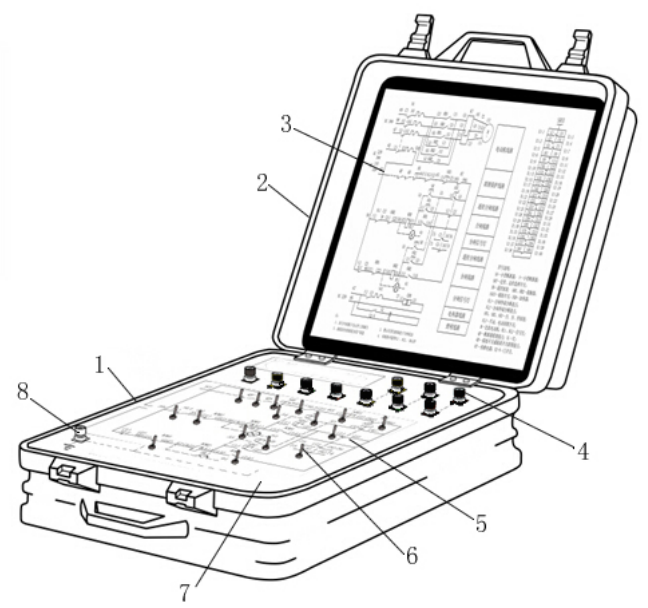

Figure1: Equipment structure

1 box, 2 cover, 3 actual wiring diagram of secondary circuit in the operation system, 4 blocks, 5 secondary circuit diagram of the operation system, 6 luminous rocker switch, 7 control panel, 8 air switch, 9 terminals row I, 10 terminal row II.

\section{Installation wiring diagram}

A, back folded view - unfold the structure of the screen on the wiring installation diagram. On the back of the screen is installed instrument panel, control switches, signal equipment and relay; on the side of the screen is installed terminal block; on the back or side of the top screen is installed small bus, fuses, additional resistance, knife switch, bell and buzzer, etc.

$\mathrm{B}$, general roles for arranging devices on the screen: on the top is relay, then intermediate relay, time relay and the lower part is relay that often needs debugging (direction, differential, reclosing, etc.), and the bottom is signal relay, connecting pieces and a light plate, light, button, switch and other controls.

$\mathrm{C}$, secondary devices on the protection and control panel diagram are all numbered from the left to right and from the up to down and marked with text symbols; text symbols are accordant with the folded view and schematic; on the side of the screen diagram lists the devices diagram (where the device's number, symbol, name, type, technical parameters, quantity, etc. are noted); for example, on the back of the screen is installed devices (such as resistors and fuses, etc.) is noted in the remarks column of the device list.

D. on the installation wearing diagram that represents secondary device - the rear-screen wearing diagram, the left and right orientation of devices is on the contrary with the screen layout diagram (rear view); draw the invisible secondary equipment contour at the rear with a dotted line; 
draw out the terminal from the inner device (screw), shown by a small circle with number of the terminal.

As shown in Fig. 2:

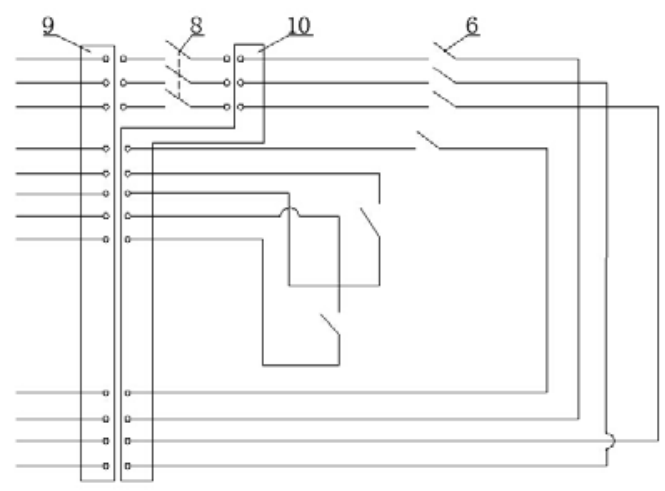

Figure 2 installation wiring diagram

\section{Method of implementation}

Secondary circuit fault clearing in electrical equipment is always a basic skill for electrical staff to grasp and also an important part in stuff training, evaluation, competition and other projects. Currently, training process for secondary circuit fault clearing of operating system is started from setting man-made faults and then requiring the trained to find out and clear the faults. Faults are traditionally set specifically by pasting tap as insulation on contact point of corresponding fault contact terminal and then reinstall it as the fault. This method takes much effort and time and frequent disassembly of wires may lead to loose screws on the contact, resulting in exposed wires and the system unable to get power support. This can only fixed by changing contactors and the process is time-consuming. In addition, since many nodes on the main loop of the operating system are crimped or plugged in, it is impossible to set man-made faults there. Much time would be wasted on theoretical analysis and schematic explanations as no actual test items is available for students to do measurement. Therefore, many faults finding can not be trained in the process of teaching and thus the training effect is not ideal.

In order to provide a structure design to solve the above problems, this paper proposes a more reasonable and time-saving secondary circuit fault finding training equipment of operating system that is suitable for a series of electrical equipment with operation systems.

On the box, cover and the top of the box is set control panel. The control panel is characterized by printed schematic of the secondary circuit of operation system; connecting nodes of corresponding electrical components of control panel on the schematic of secondary circuit in the operating system are equipped with luminous rocker switch; a terminal block I is set in the inlet position of the box; corresponding terminal blocks II with terminal block I is set inside the box; in the secondary circuit operation system available for training of outer training box, all connecting nodes of electrical components are broken and connected with wires respectively; all wires are firstly connected with terminal block I and then with terminal block II, wire from which then connected with terminates of corresponding luminous rocker switch; the wire connected with power supply line is equipped with air switch when introduced through terminal block I and terminal blocks II; on the side of the inner box is printed the actual wiring diagram of secondary circuit in the operation system.

In the above discussed secondary circuit fault finding training equipment of operating system, several terminals are set on the control penal in case that the trainer need to user temporary wiring when setting faults. In the above discussed secondary circuit fault finding training equipment of operating system, aluminum alloy shell is adopted as the body of the box and shell of the cover; inside the aluminum alloy shell is set an electrically insulating foam layer. In the above discussed 
secondary circuit fault finding training equipment of operating system, the control panel is made of high voltage electrical insulation board.

1) By controlling the on-off of all aluminum rocker switches on the control penal to control the connecting nodes of electrical components in the secondary circuit operating system available for students to do exercises outside the training box, so as to complete fault setting conveniently and efficiently without opening the box chamber repeatedly.

2) Arranging the wire inside the box through terminal block I and terminal blocks II to make the line clear, make it possible to set originally unavailable faults setting and thus increase the quality of training and teaching and at the same time make maintenance easier.

3) An air switch is set on the wire connected with the power line of secondary circuit operating system when piercing through the terminal block I and terminal blocks II. Turning off the air switch can shut down the power of entire secondary circuit operating system and thus ensure the electrical safety of entire box.

4) The actual wiring diagram of secondary circuit on the cover is available for trainers to take references efficiently..

5) The diagram is in accordant with the actual wiring of electrical devices and thus make it more visualized and intuitive for the trained to understand and grasp the method more efficiently of secondary circuit fault clearing.

\section{Conclusion}

Various protection measures have been taken to guarantee the personal safety during the actual usage of this product. PLC control circuits are also added to realize the intelligent setting of failures, thus diversifying the teaching models, training models and examination models. Most importantly, it can enhance working efficiency and working quality, facilitate the setting of secondary circuit failure and prolong the service life of equipment.

\section{References}

[1] Ou Zhirong. On the Problems in Substation Equipment Maintenance and Repair of Power Supply Companies and the Measures [J]. Technology and Market, 2013 (08).

[2] Wei Sile. The Problems in Substation Equipment Maintenance and Repair and Countermeasures [J]. China High Technology Enterprises, 2014 (03).

[3] Li Zibin. On the Problems in Substation Equipment Maintenance and Repair and Measures [J].Chinese E-Commerce, 2014 (05).

[4] Guo Jing, Ma Zhiying, Lei Qing. Optimizing deign of Cam Mechanism in Spring Operating Mechanism of Vacuum Circuit Breaker II. High-voltage electrical appliances, 2004,40 (3): 420-423.

[5] Ma Jie. Carry out Equipment Condition-based Maintenance and Ensure the Safe Operation of Power Grid[J].Sci-Tech Information Development \& Economy. 